\title{
Discourse Matrix: A Practical Tool for ESL Writing Teachers
}

\author{
Ardiss Mackie \\ Chris Bullock
}

The purpose of this article is to demonstrate how a technique involving contrastive rhetoric can help writing teachers and their students uncover and improve the overall rhetorical patterns in student writing. Contrastive rhetoric is a theory developed by Kaplan (1966), who argued that rhetorical patterns, like logic, vary from culture to culture. The discourse matrix outlined by Coe (1988), allows con- trastive rhetoric a practical application in the ESL classroom. The matrix enables both teacher and student to pinpoint areas in writing where the rhetorical pattern may not follow a typical English pattern. As well, the matrix can be used for revision. The article demonstrates the use of the matrix through examples taken from both English and ESL writers.

Every ESL teacher faces on a regular basis students' problems with grammar and other sentence level difficulties. These difficulties are addressed through numerous articles and books. However, an almost equally common problem seems much harder to find resources for. This is the problem of writing which has sentences which may or may not be grammatically effective but in any case whose overall logic seems quite unfathomable.

One explanation for these problems in overall logic is provided by the theory of contrastive rhetoric. The concept of contrastive rhetoric was developed by Robert Kaplan, beginning in his 1966 article, "Cultural Thought Patterns in Inter-Cultural Education." Kaplan argues that rhetoric, in the sense of the organization of overall discourse patterns, "varies from culture to culture and even from time to time within a given culture." (Kaplan, 1966, 2)

Kaplan analyzes samples of Semitic, Oriental, Romance, Russian and English essay texts in order to show distinctly different rhetorical patterns operating. The promise of contrastive rhetoric is that it distinguishes the overall patterns of English composition from those by which the second language learner may be operating. Kaplan points out, "The foreign student who has mastered the syntax of English may still write a bad paragraph or a bad paper unless he also masters the logic of English." (15)

An objection to explaining overall discourse problems primarily by contrastive rhetoric has been made by Mohan and Lo (1985) in their article, 
"Academic Writing and Chinese Students: Transfer and Developmental Factors." They argue that, in the case of Chinese expository writing, school and government prescriptions are not much different from similar prescriptions in North America, and that these students' discourse problems more likely derive from their writing development. David Piper (1985) questions Kaplan's advocacy of introducing advanced ESL students to both the rhetorics of their own and of their target discourse forms from another angle. As a result of examining a number of studies of language processing in ESL reading, Piper concludes that Kaplan's approach-the direct teaching of contrastive rhetoric-is only one of a number of pedagogical options which the research supports. While some strands of the research support Kaplan's approach, other strands support students "writing materials in th(e) second language based on the first language culture" (36) and even the approach of "maximal immersion in . . . the language and culture to be acquired" (37).

Mohan and Lo's view that Chinese writers obey very similar rhetorical rules to English writers is contradicted by Carolyn Matalene's experiences as described in, "Contrastive Rhetoric: An American Writing Teacher in China" (Matalene, 1985). In her ESL teaching in China, Matalene found writing patterns to be strongly influened by cultural thought patterns. Mohan and Lo also neglect to refer to the substantial study by Kyoko Oi (1984) whose contrastive study of English and Japanese rhetoric and whose extensive critical literature review strongly support the premises of contrastive rhetoric. Another study contradicting Mohan and Lo's view was conducted by Carrell (1984). In her study of the effects of rhetorical organization on ESL readers, there seemed to be certain English rhetorical patterns which were more or less easily recalled among different first language groups. She concluded that although further study in this area was required, her findings pointed to "interference from the preferred native rhetorical patterns." (464) As to David Piper's case, it provides a needed corrective to a narrow definition of contrastive rhetoric, but not a repudiation of Kaplan's approach; Piper's conclusion is that "the most principled suggestion of all might be to vary classroom activities between each of the aforementioned pedagogical options" (42).

What the research seems to show is that while we should not ignore the problems that come simply from inadequacies in writing development, and while we should recognize the validity of other pedagogical approaches, there are good grounds for introducing the direct teaching of contrastive rhetoric into the ESL classroom. This being so, the next question is: How can the tool of contrastive rhetoric be best put to use? On the level of practical classroom application, the problem is that writers like Kaplan offer their conclusions about various pieces of student writing, but do not offer a systematic and accessible method for analyzing them. In 
our view, the first text to offer such a method is Richard Coe's Toward a Grammar of Passages (1988).

For those teachers interested in theory, Towards a Grammar of Passages offers chapters arguing the need for a theory of the paragraph or passage, discussing the origins of Coe's own work in Francis Christenson's generative rhetoric of the paragraph, and linking this work to the work of philosophers like Michel Foucault and composition theorists like James Berlin and Richard Ohmann. Teachers interested in practical applications, though, will be most interested in Chapters Three and Four. Chapter Three outlines Coe's discourse matrix, a reworking of Christenson in the form of a graphical representation of levels of generality in paragraphs, while Chapter Four examines the applications of the matrix by detailing several specific studies conducted by Coe's students in China and Canada.

A most important source for Coe's discourse matrix is Francis Christenson's "A Generative Rhetoric of the Paragraph." In this article, first published in 1965, Christenson argues that just as a sentence consists of a series of elements related to each other by a structure of coordination and subordination, so a paragraph can be defined as "a sequence of structurally related sentences . . . related to one another by coordination and subordination" $(1979,79)$. Christenson is thus offering a way of analyzing paragraphs in terms of levels of generality, and he also discusses some of the devices (including particular expressions, repetition of structures) that serve to indicate relationships of coordination and subordination. However, though Christenson's article is seminal, his procedure of representing numbered sentences at different points of indentation is very cumbersome for a teacher or student to reproduce. What Coe has done is to replace this cumbersome procedure with a graphical procedure in which numbers stand in for the numbered sentences and lines moving down, up, or on the level indicate relationships between sentences of subordination, superordination or coordination respectively.

Coe describes the technique for constructing a discourse matrix as follows:

one divides the test into units, usually T-units, although the analysis can also be done with larger or smaller units (whole sentences or clauses). These units are then numbered consecutively, and each Tunit is analyzed to determine where it connects to the discourse (usually-but not always-to the preceding T-unit) and whether it is subordinate, coordinate or superordinate. Although it is not strictly necessary, naming the relationship more specifically . . . often aids the analysis. To draw a matrix, one represents each $\mathrm{T}$-unit by a numbered circle, represents that T-unit's level of generality by the relative placement of the circle on the page, and represents the logical (or "modifying") relationship with a line connecting the numbered circle to another (34 to 35 ). 
Before demonstrating this procedure, two comments seem necessary. First, we should notice that the precise unit of analysis is not of prime importance in the drawing of a discourse matrix. In "A Generative Rhetoric of the Paragraph," the sentence is Christenson's unit of analysis, while in Grammar, Coe's is the T-unit, defined by its originator, Kellogg Hunt, as a "main clause plus any subordinate clause or nonclausal structure that is attached to or embedded in it" $(1970,4)$. In our demonstrations we will follow Coe's example, since the use of T-units allows a more sensitive analysis of the problem of excessive coordination found in some ESL writing. However neither we nor Coe are using T-units in order to address Hunt's particular area of concern: the assessment of syntactic complexity. The desirability or otherwise of such complexity is not an issue which the use of a discourse matrix directly addresses.

Second, we should emphasize that what Coe calls "naming the relationship" between two T-units is indeed immeasurably helpful in the often quite tricky task of establishing relationships between T-units based on levels of generality. "Naming the relationship" is a rather ambiguous formulation; what it actually means is recognizing that various particular rhetorical operations almost invariably result in relationships of coordination, subordination or superordination. Thus as Coe, taking his material from Sandra Stotsky, points out, a T-unit presented as a contrast, a contradiction, a conjoining or a repetition will likely have a relation of coordination to the previous T-unit; a T-unit presented as a definition, an example, a reason, a deduction, an explanation, or a qualification will likely have a relationship of subordination to a T-unit at a higher level of generality; and a T-unit presented as a conclusion, a generalization from, or a comment on, will likely have a superordinate relationship with the previous T-unit (Stotsky, 1983, 441; cited in Coe, 32).

Let us imagine we are following Coe's procedure in order to draw a discourse matrix for the following brief paragraph:

Many Canadians strongly oppose capital punishment but just as many are in support of it. For example, a recent poll showed 55\% of Canadians in support of its reimposition. My casual conversations with individuals in the street suggest a quite even divide on the issue.

First, we divide the text into T-units, noting that the first sentence has two such units and the next two sentences form one T-unit each: 1. Many Canadians. . . 2. but just as many. . . 3. For example. . . 4. My casual conversations. ... .

What is the relationship of the first two T-units? They are clearly on the same level of generality, being alternate framings of the same concept: having an attitude to capital punishment. Furthermore, as alternate framings of the same concept, they are clearly connected. Thus to follow Coe's 
procedure, we would put them on the same level and join them with a line, either above or below, to indicate their connection:

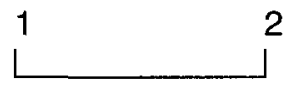

What about the third and fourth T-units? They are clearly examples of the statement affirmed in the first two T-units and thus (as Coe's listing indicates) are clearly subordinate to those units and connected to them. They are thus put at a lower level than the first two units, and then joined to those units and to each other to indicate their status as sequential examples;

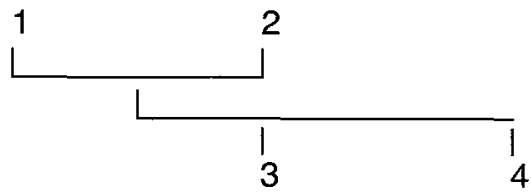

In his book, Coe uses the matrix simply to identify patterns in Chinese and Farsi writing. We believe it is more helpful for ESL teachers to see the discourse matrix as having the function of identifying desirable models for revision as well as simply being used to identify cultural rhetorical patterns in students' own writing. The most obvious model to guide revision is the typical English academic paragraph.

Here is a paragraph of typical basic English academic writing, together with its accompanying matrix:

1. The third reason to value an arts degree is the mental flexibility it gives the student. 2. By mental flexibility is meant the ability to respond creatively to the different needs of different people and of changing situations. 3. Arts graduates have done very well in public relations. 4 . They occupy many editorial positions in publishing and the media. 5. Some arts graduates have also done very well in sales positions. 6 . All these kinds of work require recognizing the different needs of different audiences.

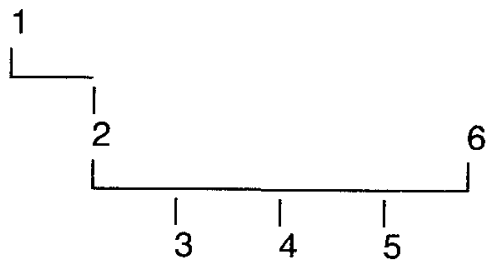

As we can see from the matrix, the typical basic academic paragraph has three levels of generality: topic sentence generalization (1), examples and details $(3,4,5)$, and explanations linking examples of details to the 
topic sentence generalizations $(2,6)$. To construct the matrix for this paragraph, we start by allowing T-unit 1 , the topic sentence, to stand at the highest level of generality. Next, we draw a line under T-unit 1 to T-unit 2 to indicate that $\mathrm{T}$-unit 2 is connected to, but at a lower level of generality than, the topic sentence. In other words, the definition of mental flexibility (T-unit 2 ) is connected to, but more specific than, a topic sentence about the value of mental flexibility. The line connecting T-unit 2 to T-unit 3 tells us that $\mathrm{T}$-unit 3 is connected to, but more specific than T-units 1 and 2. T-units 3, 4, and 5 are connected and at the same level of generality because they are sequential examples of areas of employment that arts degree graduates hold. These examples are of more specific content than the topic sentence or the definition. The final $\mathrm{T}$-unit, 6 , is connected to $\mathrm{T}$-unit 5 because it summarizes the examples at the second level of generality, the same level as the definition. So the line must go up from T-unit 5 to T-unit 6.

Now to demonstrate the other function of the matrix, let us analyze some examples of student writing. The first sample is from an Arabicspeaking student, writing in response to an invitation to discuss no smoking laws.

1. The last reason for making no-smoking laws is that the adverse social effects. 2. Smokers who have children encourage their children to smoke. 3. As a result, smoking disturbs their children-growth. 4 . And it declines their motivation for studying. 5. Another example is one of the family doesn't like smoking, they will have troubles to live together 6 . and perhaps, it causes to divorce. 7 . Moreover, smokers have troubles to deal with non-smoker guests in their homes. 8 . To conclude, smokers have many difficulties to live both in their home and in their society.

The first step in the procedure is to number the T-units, as in the paragraph above. The second step is to identify the levels of subordination. When we analyze the text in this way, we see that there are three levels of generality: T-units 1 and 8 are establishing general statements about social effects; T-units 2, 5, 6, and 7 are examples of particular social effects; T-units 3 and 4 modify T-unit 2. Put into graphic form, these relationships would look like this:

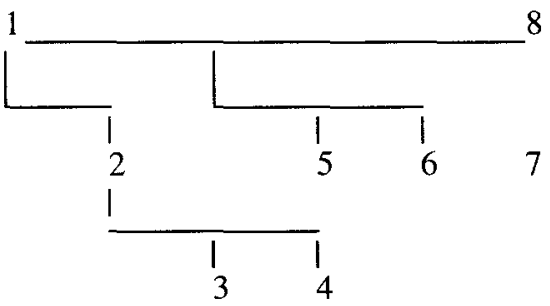


The second stage of the process is to identify any distinctive features of the paragraph as graphically illustrated and to compare the paragraph in its discourse matrix form with the desired rhetorical model in English composition. This comparison then forms the basis for suggesting revisions.

In the sample paragraph we are discussing, the problem is the proliferation of examples at the same level of generality in T-units 5 to 7 . This problem can be related to the tendency of Arabic writing, as noted by Kaplan (1966), to accumulate parallel or coordinate constructions.

This analysis of the paragraph suggests two possibilities for revision. As one possibility, the student could narrow the paragraph topic and expand T-units 2,3 , and 4 . The other possibility is for the student to omit T-units 6 and 7 and instead develop two or more T-units subordinate to T-unit 5. As the following illustrations show, this revision would create the three levels of hierarchy characteristics of the basic academic essay in English.
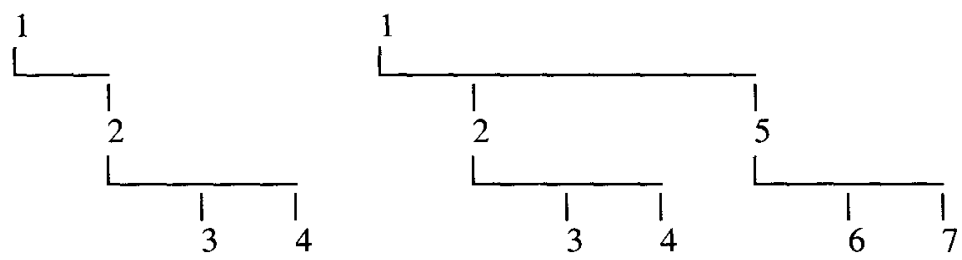

The second example, followed by its corresponding matrix, comes from a Chinese student writing a paragraph on the topic. Does mental health affect physical health?:

1. No, I don't think so. 2. I think physical health can help people keep their strong health and develop their mental health. 3. I think people should spend one hour to play some sports every day. 4 . If you hope to have a healthy body, you must to play some sports. 5 . Because your body consist of many parts, sports will take these parts moving. 6. I think only sports can help people have a healthy body. 7. People's mental health is same as physical health, 8. all need sports. 9. Sports make relax for people. 10. At that time people have not stress, only have happy and a little tired. 1

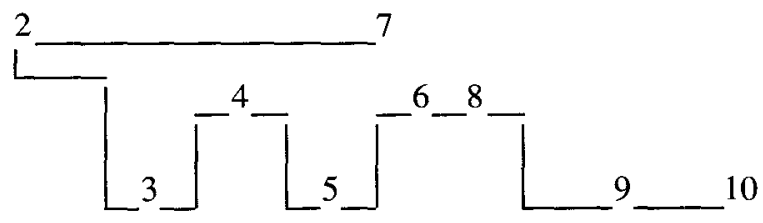

Clearly this student needs work on grammar in individual sentences. However, the overall discourse pattern of the paragraph also creates dif- 
ficulties for the English reader. The matrix shows three levels of subordination developed like a see-saw through $10 \mathrm{~T}$-units. The first T-unit is disconnected from the rest of the text. This disconnection underscores earlier research which characterized Chinese writing as having an opening statement contrary to the basic theme (Oi, 1984, 61).

Rearranging the T-units so that they follow a more linear pattern would improve her writing, although even then certain statements would have to be dropped to allow a single thesis to develop. Such a revision is, however, helped by the fact that the student's writing already contains three levels of generality.

In helping the student revise, we could emphasize that her main idea is the idea that achieving physical health can help people keep their mental health, and that the main way of achieving physical health that she describes is the practice of sports. We could then suggest that she reorganize the paragraph to focus on these elements. These suggestions for the reorganization of her discourse would go along with, and make more meaningful, suggestions for grammatical improvement. The paragraph that might result from these suggestions:

1. I think physical health can help people keep and develop their mental health. 2. Only sports can help people have a healthy body. 3. I think people should spend one hour to play some sports every day. 4. Sports make people relaxed. 5. Doing sports, people lose stress and become happy and a little tired.

Or in matrix form:

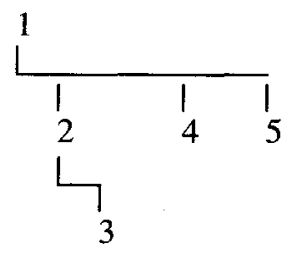

How can the teacher be sure that the revisions she has made to paragraphs and matrixes reflect the student's intended focus and meaning? We suggest that revisions be verified in consultation with the student. In this way, teachers and students can be assured that the intentions of student writing are correctly represented.

In conclusion, we hope we have shown by these examples that the matrix is a practical tool for helping ESL writers with rhetorical and logical problems. However, it is important to realize that using the discourse matrix in contrastive rhetoric has certain limitations. As we point out in a review of Coe's book, "the matrix cannot be consistently relied upon to uncover characteristics of non-linear rhetoric that relate to content" (Bul- 
lock and Mackie, 1988, 7). For example, we noted that the matrix failed to identify the use of proverbs at the end of a text typical of Arabic discourse as Kaplan $(1966,8)$ discussed. We should also note that the discourse matrix would be misused if it led us to ignore the validity of the logic and rhetoric of cultures other than our own. Matalene $(1984,806)$ makes this point very strongly:

Those who have done their years of memorizing and have mastered the Chinese tradition of rhetoric and logic have done more than become literate. They have learned how to behave, what to say, and how to say it. They have gained entrance to the beauty, often the tragic beauty, of a centuries old tradition and the right and the privilege to contribute to it. Our responsibility is surely to try to understand and appreciate, to admit the relativity of our own rhetoric and to realize that logics different from our own are not necessarily illogical.

If properly used, however, the discourse matrix can pinpoint the structures of basic English academic writing and outline for students the difference between those and the rhetorical structures with which they are operating. The importance of Toward a Grammar of Passages is that it makes contrastive rhetoric a tool which the ESL teacher can put to practical use in the classroom.

\section{REFERENCES}

Bullock, Chris and Ardiss Mackie. (1988). "A Review of Toward a Grammar of Passages." Inkshed, 7(3), 5-8.

Carrell, Patricia L. (1984). "The Effects of Rhetorical Organization on ESL Readers." TESOL Quarterly, 18(3), 441-469.

Christenson, Francis (1978) Notes Towards a New Rhetoric. Second edn. New York: Harper and Row.

Coe, Richard M. (1988). Toward a Grammar of Passages. USA: Southern Illinois UP.

Hunt, Kellogg W. (1970). "Syntactic Maturity in School Children and Adults." Monographs of the Society for Research in Child Development, 35.

Kaplan, Robert B. (1966). "Cultural Thought Patterns in Inter-Cultural Education." Language Learning, 18, 1-20.

Matalene, Carolyn (1985) "Contrastive Rhetoric: An American Writing Teacher in China. College English, 47, 789-808.

Mohan, Bernard A. and Winnie Au-Yeung Lo. (1985). "Academic Writing and Chinese Students: Transfer and Developmental Factors." TESOL Quaterly 19 (3), 515-534. 
Oi, Kyoko. (1984). Cross Cultural Differences in Rhetorical Patterning: A Study of Japanese and English. University Microfilms International.

Piper, David. (1985). "Contrastive Rhetoric and Reading in a Second Language: Theoretical Perspectives on Classroom Practice." Canadian Modern Language Review, 42 (1), 34-43.

Stotsky, Sandra. (1983). "Types of Lexical Cohesion in Expository Writing: Implications for Developing the Vocabulary of Academic Discourse." College Composition and Communication, 34, 430-446.

\section{THE AUTHORS}

Chris Bullock is Professor of English at the University of Alberta, and joint author of A Guide to Marxist Literary Criticism for the Student of English (1980) and Essay Writing for Canadian Students (1985). He specializes in teaching the theory and practice of rhetoric and composition.

Ardiss Mackie is Assistant Director of the English Language Program in the Faculty of Extension, University of Alberta. She is a graduate of the TESL Centre, Concordia University and joint author of Suspicious Minds (in press), an ESL listening textbook. 